\title{
«Los niños aquí van solos al colegio». Maternidad en las encrucijadas de la migración de peruanas en Aichi, Japón
}

\author{
Akemi Matsumura \\ Universidad de Nagoya \\ amatsumura@pucp.pe
}

RESUMEN

Luego de más de veinte años de las primeras migraciones masivas de peruanos a Japón, las mujeres peruanas enfrentan retos al criar a la segunda generación de migrantes en este país. En este contexto, esta investigación buscó responder a la pregunta de cómo se redefine la maternidad. Los recorridos migratorios de las mujeres, su relación entre la casa (households), trabajo y escuela son claves en esta redefinición. Así, pues, este artículo plantea que sus motivaciones migratorias, los roles de género como madres y esposas y la crianza diferenciada de sus hijos son las principales aristas de los cuestionamientos sobre sus conceptos de maternidad como madre guía. Este artículo está basado en una investigación cualitativa para la cual se llevó a cabo un seguimiento etnográfico de mujeres peruanas madres que viven en la prefectura de Aichi, Japón, entre 2016 y 2017.

Palabras claves: maternidad, migración, Japón, peruanas, género. 


\section{«Kids here go to school alone». Motherhood in the crossroads of Peruvian migrants in Aichi, Japan}

ABSTRACT

After more than 20 years of the first massive migrations of Peruvians to Japan, Peruvian women face challenges in raising the second generation of migrants in this country. In this context, this research tries to answer how these women re-define their motherhood. The migratory routes of women, their relationship between households, work and school are key in this re-conceptualization. Thus, this article shows that their migratory motivations, their gender roles as mothers and wives, and the different upbringings of their children are the main factors that question their concepts of motherhood as guiding mothers. This paper is based on a qualitative research, elaborated under an ethnographic methodology, carrying out fieldwork with Peruvian mothers living in Aichi prefecture, Japan between 2016 and 2017.

Keywords: motherhood, migration, Japan, Peruvian women, gender. 
A partir de 1990, luego de la revisión del acta de inmigración que facilitaba visas para descendientes japoneses, Japón se convirtió en uno de los destinos más importantes para los peruanos (INEI, 2008) ${ }^{1}$. Con relación a este proceso, se han realizado importantes investigaciones acerca de la adaptación de peruanos en Japón, su situación laboral, la inserción de sus hijos en la escuela, movilidad y exclusión social, etc. (Lagones, 2016; Melgar Tisoc, 2014; Moorehead, 2007; Nakamura, 2011). Sin embargo, no ha existido un enfoque de género que muestre cómo hombres, mujeres y minorías del colectivo LGTBIQ experimentan el proceso migratorio de forma diversa. Este artículo plantea aproximarse a la experiencia migratoria de mujeres peruanas madres en Japón como parte de la investigación que realicé entre 2016 y 2017 para mi tesis de maestría.

Desde el siglo $\mathrm{XX}^{2}$, tanto a las últimas generaciones de madres como a las actuales se atribuye la responsabilidad del bienestar emocional de la familia, traducido en la buena socialización de los menores (Palomar, 2005; Weiss, 1978). Es así que hablamos de reproducción social, ya que buena maternidad y buena crianza significan que los nuevos sujetos llenen las expectativas culturales en términos de desarrollo emocional, cumplimiento de sus roles en la sociedad, etc. (Weisner, 2015).

Hasta hace pocas décadas, las generaciones pasadas, para asegurarse de que los hijos no cayeran en dinámicas antisociales, utilizaban estrategias de represión a través de restricciones, castigo físico, psicológico, etc. La madre no era una confidente, sino un árbitro. Hoy en día, este tipo de maternidad se encuentra en constante cambio propiciado por transformaciones en los roles de género: el

A través de la falsificación de documentos, no solo descendientes japoneses pudieron acceder a visas de trabajador o residente en Japón, sino también peruanos no descendientes.

2 Desde el siglo XX, la presión por ser una «buena madre» se incrementó desde que los expertos empezaron a girar su enfoque de la profesionalización de la maternidad a la importancia de esta en el buen desarrollo de los infantes (Weiss, 1978, en Palomar, 2005). 
ingreso masivo de la mujer a las universidades y al mercado laboral, las nuevas tendencias en pedagogía y psicología, etc. (Raffaeta, 2016). Por ello, existe un grupo más extenso de mujeres con acceso a conocimiento académico, especialmente desde la psicología y la pedagogía, lo que hace que los conceptos sobre maternidad, aprendidos por tradición y crianza, sean cuestionados. Hoy en día, la maternidad está más orientada a ser una guía más que un arbitraje. La represión no es la estrategia más aceptada: más bien se encuentra en constante cuestionamiento y se piensa más en términos de diálogo. Esto trae nuevas exigencias para lo que es considerado «buena maternidad»: el uso de conocimiento académico, junto con las nuevas exigencias de ser mujer, como ser económicamente independiente, tener una carrera profesional y ser madre al mismo tiempo. Esto representa nuevos retos: ¿cómo ser una madre comprensiva en el tiempo que te queda luego de trabajar?

La migración peruana en Japón tiene más de veinticinco años. Jakeline Lagones y Robert Moorehead, entre otros, han mostrado que el principal motivo para permanecer en Japón ha sido la formación de familias (específicamente convertirse en padres), en comparación con los que retornaron al Perú, quienes mayormente lo hicieron siendo solteros (Lagones, 2016; Moorehead, 2010). Es por eso que ya se puede hablar de una segunda y tercera generación de peruanos en el Japón. Sobre estos padres migrantes se ha escrito de forma general, en especial sobre sus limitaciones en la escuela y cómo se forma un concepto de otredad frente a la sociedad japonesa.

A pesar de estos aportes, no se ha tomado en cuenta la participación diferenciada de hombres y mujeres en el proceso de crianza de esta segunda generación. Las mujeres, independientemente de tener un trabajo remunerado o no remunerado, son las principales encargadas de asistir a las reuniones de padres de familia en el colegio, llevar a los niños y niñas al hospital, ayudarlos con las tareas escolares, entre otras labores de crianza. Esta problemática coincide con el Informe mundial de mujeres trabajadoras 2015 (Samman, Prestel-Marshall, Jones, Bhatkal, Melamed, Stavropoulou y Wallace, 2016). Por eso decidí entrevistar y observar mujeres ${ }^{3}$ : por tener experiencias más cercanas a la crianza de estos niños migrantes de primera o segunda generación. Tomando este contexto como base, la investigación tiene a la maternidad como concepto de discusión central. En este artículo, a partir de la revisión de casos etnográficos, exploro cómo el

Adicionalmente entrevisté a un par de esposos de estas mujeres peruanas en la prefectura de Aichi. 
proceso migratorio ha influido en los conceptos y el ejercicio de la maternidad en peruanas en Japón, y en especial, alrededor de sus motivaciones migratorias, sus roles de género como madres y esposas, y la crianza diferenciada. Por último, busco una reflexión sobre la maternidad en contextos de migración, pero también a la luz de los cambios globales actuales.

\section{Sobre la investigación}

Mi trabajo de campo se llevó a cabo en la prefectura de Aichi, en especial en dos ciudades: Nagoya e Inuyama, durante nueve meses. Aichi, en la zona central de Japón, fue escogida debido a que es una zona industrial que tiene un alto número de trabajadores migrantes y a que ha sido la prefectura con mayor número de migrantes peruanos en todo Japón (Ministerio de Justicia de Japón, 2013, en Lagones, 2016). También escogí las ciudades de Nagoya e Inuyama para obtener una muestra relativamente diversa de mujeres peruanas en Aichi. Esto debido a que Nagoya es la capital de esta prefectura y la tercera ciudad más grande de Japón. En ella existe un consulado peruano y conecta con varias ciudades pequeñas (Toyota, Kasugai e Inuyama, entre otras) y medianas, en cuyas fábricas trabajan muchos peruanos. Las mujeres que entrevisté en Nagoya por lo general ya no trabajaban en fábricas, sino que estaban casadas con ciudadanos japoneses o tenían algún otro tipo de ocupación o negocio. Por su parte, Inuyama es una pequeña ciudad con varias fábricas y mayor concentración de peruanos. Sin embargo, es importante anotar que en ambas ciudades (y en todo Japón), luego de la crisis económica mundial de 2008, el número de peruanos se redujo, ya que el gobierno otorgó incentivos económicos para promover su regreso al Perú (Ministerio de Justicia de Japón, 2013, en Lagones, 2016). En ambas ciudades - y sobre todo en Nagoya-, los peruanos no estaban «guetificados» en vecindarios o distritos sino distanciados geográficamente.

\section{MUJERES PERUANAS EN JAPÓN: SUS MOTIVACIONES PARA MIGRAR Y SU ACTITUD HACIA JAPÓN}

Las razones para migrar han sido extensamente estudiadas y es aceptado que son, en primer lugar, intereses económicos, y en segundo, la reunificación familiar en la cual los miembros del lugar de origen se unen con los de destino (Hernández, 2007). En los estudios sobre mujeres migrantes (especialmente en Europa y Estados Unidos) está muy extendida la suposición de que las mujeres 
migran mayormente para encontrarse con sus parejas varones, quienes son los principales sujetos económicos, es decir, los que migran para entrar al mercado laboral y sostener económicamente una o más familias (Fritzs, Kummels y Stefan, 2013). Sin embargo, en muchos de los casos que registré se trataba de mujeres que habían migrado solteras, en algunos casos con un hijo o acompañadas por otras contemporáneas solteras. Muchas de ellas estaban empleadas en un trabajo profesional o técnico, como profesora de inicial, secretaria, oficinista, etc. Por medio de redes sociales, fueron informadas de que en Japón podrían recibir mejor remuneración por un trabajo no profesional, en comparación con el trabajo que ejercían en ese entonces en el Perú.

Un factor menos estudiado que los anteriores es el deseo de conocer nuevos espacios y tener un tipo de vida diferente del que conocieron en el Perú (Hernández, 2006). Muchas de ellas sabían muy poco de Japón, pero sus expectativas estaban basadas en la imagen de Japón como un país desarrollado, por lo mismo superior a todo lo que habían conocido en el Perú. Este imaginario estaba especialmente basado en su deseo de tener una mejor calidad de vida reflejado en dos aspectos principales: un mayor acceso al consumo y a un sistema de seguro social del Estado. Un cuarto factor de su motivación era el acceso a la visa de trabajador que Japón ofrecía (Lagones, 2016). Para ilustrar estas motivaciones, utilizaré los casos de Luz y Patricia.

En Lima, Luz empezó a estudiar corte y confección, carrera que no culminó, para luego trabajar como profesora de manualidades. En 1993, una amiga le propuso que la acompañara a trabajar a Japón. Por medio de un contratista chino, lograron conseguir un contrato en una fábrica. Luz viajó con esta amiga y su sobrina, quien regresó pocos meses después, ya que no se acostumbró a estar a la distancia de su familia y su novio. Luz también regresó al Perú un año después, ya que sentía que no se acostumbraba a la vida en Japón. Sin embargo, luego de unos meses decidió que quería regresar, pues no se acostumbraba a vivir en Lima nuevamente. Trabajando en una fábrica en Japón podría tener más independencia económica y social (podría vivir fuera de la casa de sus padres). Pero, sobre todo, en el Perú no se acostumbraba a la informalidad. Un amigo japonés le envió una carta de invitación para que pudiera entrar al país por quince días. Luego de eso, Luz vivió en Japón por varios años ilegalmente, hasta que se casó con su actual esposo, un ciudadano japonés. En ese año, tenía dos hijas de veintidós y veinte años y vendía comida peruana fuera del consulado de Perú en Nagoya. Nunca pensó seriamente en retornar al Perú, especialmente por sus hijas y su esposo, quienes solo veían al Perú como un destino turístico y para la visita a los familiares. 
En el caso de Patricia, ella inició varios cursos y carreras que no pudo culminar (francés, contabilidad, inglés, psicología, arte, etc.). Ella deseaba estudiar psicología, pero su padre le impuso que estudiara contabilidad. Por medio de una amiga, quien le dijo que en Japón podían tener trabajos bien pagados, contactó a un contratista, quien era un intermediario entre ellas y un empleador en Japón. A través de un conocido de la familia que era descendiente de japoneses, Patricia falsificó documentos y pudo obtener la visa. Luego de llegar a Japón, trabajó en una fábrica por un año y le empresa no quiso renovarle el contrato, por lo tanto, su visa expiró. Se convirtió en ilegal por varios años. Los primeros de estos fueron tiempos duros, en los cuales incluso tuvo que dormir en baños públicos. «En un momento, mi estado legal salía como "criminal". Eso fue chocante», comenta Patricia.

Meses después, consiguió un trabajo en un campo de golf donde la recibieron aun siendo ilegal. Ahí pudo socializar con muchos clientes japoneses, ya que tenía que acompañarlos en la cancha de golf, hablar con ellos y asistirlos. Trabajó en esto durante seis años, en los que aprendió japonés en un nivel avanzado, excepto en la escritura. Su situación legal solo cambió cuando se casó con un hombre brasileño con visa de residente con el cual tuvo dos hijos. Se separaron cuando estaba embarazada de su segunda hija. Actualmente trabaja de traductora de castellano, inglés, japonés y portugués en una escuela cerca de la ciudad de Inuyama. Un año antes de que la entrevistara, había traído a su madre ilegalmente para que la ayudase con la crianza de sus hijos. Como traductora de la escuela, es conocida por muchas otras madres migrantes, a quienes ayuda no solo en la escuela, sino también con sus otros problemas en Japón: el hospital, los seguros, la policía, etc. No sueña con regresar al Perú.

En ambos casos, las entrevistadas no culminaron estudios superiores y migraron cuando eran aún solteras, acompañadas de contemporáneas. La decisión de quedarse a pesar de su situación de ilegalidad fue llevada a cabo de forma individual y no por seguir a su familia. Estos casos forman parte de una tendencia entre mis entrevistadas, quienes se caracterizan por tomar la decisión de migrar estando solteras o siendo madres solteras, por su propia decisión. Estas presentan mejores condiciones y actitudes para adaptarse a Japón en comparación con las mujeres que migraron para encontrarse o acompañar a sus familias. A continuación, explicaré este proceso diferenciado.

Debido a que estaban solteras, pudieron trabajar en puestos de atención al cliente (por ejemplo: night clubs, escuelas de golf, cuidado de ancianos, etc.), donde los horarios eran muy variables. En ellos pudieron tejer redes y aprender 
el idioma japonés, lo que significó un gran recurso para conseguir algún tipo de ascenso social - como un trabajo de traductora-, expandir sus redes sociales, casarse con un ciudadano japonés y legalizar su situación. Por lo contrario, las mujeres que llegaron siguiendo a sus parejas, padres u otros parientes, por lo general trabajaron en fábricas junto con ellos o decidieron cuidar exclusivamente a sus niños, espacios donde socializaban poco con nativos japoneses. En esta situación, se encontraron con mayores niveles de estrés. En todo caso, convertirse en madre significó también tener un connacional con el cual entablar una relación estable, garantizada por el parentesco. Esto es especialmente importante en un contexto en el cual los peruanos migrantes establecen relaciones poco duraderas y es difícil hablar de una «comunidad» de migrantes peruanos ${ }^{4}$. Así, las mujeres que migraron solteras adquirieron importantes recursos personales, como el idioma, redes y trabajos fuera de la fábrica.

Es sabido que todos los migrantes atraviesan situaciones de desventaja, como ilegalidad, pobreza, discriminación, etc. (Brettel, 2008; Yamashiro, 2013). En el caso de estas mujeres solteras, todo esto va sumado a las escasas redes de apoyo económico y emocional. A pesar de ello, permanecieron hasta la actualidad. Si bien luego de varios años la mayoría de ellas contrajo matrimonio, en sus primeros años decidieron quedarse, a pesar de su estatus ilegal, motivadas por la vida que podrían construir en Japón: independencia, acceso a diferentes niveles de consumo, seguridad para ellas como mujeres y sus familias, etc. Es decir, ya que fue su propia decisión empezar una vida como migrantes solteras en un país en donde no hablaban el idioma, utilizaron todos sus recursos (tiempo y motivación) para adaptarse. Por su parte, las mujeres que llegaron con sus familias percibieron la migración como un sacrificio para el bienestar de sus hijos o para estar cerca de sus familias. Por lo general, este grupo muestra más deseos de retornar, aunque son conscientes de la pérdida de redes sociales y de otros recursos.

El hecho de que las mujeres solteras hayan tenido más acceso a trabajos en donde han tenido mayor contacto con japoneses no es casual: responde a la demanda global de cuidado en el que las mujeres son las principales ofertantes (Anderson, 2012). Vemos, entonces, lo que autores en materia de género y migración han querido mostrar: que hombres y mujeres crean distintos tipos de redes

En mi tesis, explico dos razones de estas dinámicas: la primera, el desprestigio de los peruanos frente a los japoneses, por lo que formar guetos les reduce prestigio social (Moorehead, 2010); la segunda, que los peruanos en general provienen de una gran variedad de estratos sociales, geografías, contextos culturales y no han logrado construir una identidad peruana unificadora en el lugar de destino. 
de contacto (Wiltshire, 1992; Mahler y Pessar, 2006). En el caso de los peruanos en Japón, con pocas excepciones, los hombres se dedican al trabajo en fábricas. En el caso de las mujeres, la mayoría de las que reciben remuneración trabajan también en fábricas; sin embargo, es más recurrente que consigan trabajos en atención al cliente y cuidado. El trabajo en la fábrica es físicamente demandante, y en varios casos arriesgado, ya que se sabe de numerosos accidentes. Los hombres son considerados más aptos para ello, lo que perpetúa la violencia hacia ellas al considerarlas menos hábiles. Como dice el esposo de Jackelyn: «si usted quiere saber cómo la pasan las mujeres en Japón, yo creo que la pasan peor, en la fábrica las tratan mal».

Basándome en mis datos, considero que la situación laboral de las mujeres peruanas en Japón ha cambiado en mayor medida que la de los hombres luego de más de veinte años de la revisión del acta de inmigración. Hechos como estos aún no han sido lo suficientemente estudiados. Esto influye en la forma en que ejercieron su maternidad ya que, al cambiar sus espacios laborales, también cambia el tipo de recursos con los que cuentan para ser madres. Más importante aún es que estos espacios laborales influyen no solo en su ejercicio de maternidad, sino también en qué entienden por tal y cómo lo cuestionan (Fuller y Viveros, 2001).

\section{CASARSE, TRABAJAR Y TENER HIJOS: RELACIÓN Y CAMBIOS EN LOS ROLES DE GÉNERO}

La relación entre contraer matrimonio y la actividad laboral ha sido tradicionalmente simple (Olavarría, 2017). Tanto en el Perú, luego del período colonial, como en Japón, casarse significaba dejar un trabajo remunerado por uno no remunerado en casa y ocuparse de los intereses del esposo y del cuidado de los niños. En ambos países, esta figura ha cambiado de formas muy diversas. En ambos, las mujeres ingresaron a las universidades de forma masiva durante el siglo XX y se ha mantenido un paradigma de mujer moderna con estudios superiores y económicamente independiente. En el Perú podemos hablar de una gran diversidad de situaciones que responden a otras variables, como clases sociales, nivel educativo, etnicidad, geografía, etc. ${ }^{5}$.

Japón significa un caso excepcional, ya que es uno de los escasos países en donde las parejas casadas tienen impuestos más altos. En este sentido, cuando

Por ejemplo, en los últimos años se ha venido estudiando que el número de mujeres de zonas rurales con estudios superiores se ha incrementado (Ames, 2014). 
ambas partes tienen trabajos remunerados, sus impuestos se elevan considerablemente, así como todos los servicios del Estado: seguro social, escuela, hospital, etc. (Nakamoto, 2014). En muchos casos, esto desmotiva a las mujeres a continuar con su vida profesional o laboral para dedicarse al cuidado de los niños, lo que en este país puede ser económicamente más ventajoso que trabajar largas horas diarias y contratar a una niñera. Por otro lado, el gobierno está buscando incluir a las mujeres en el mercado laboral (sin mucho éxito), dados sus considerables problemas de decrecimiento y envejecimiento poblacional. Así, las mujeres enfrentan grandes retos entre la vida familiar y laboral (Wingfield-Hayes, 2013).

En el caso de las mujeres peruanas entrevistadas, contraer matrimonio en Japón significó un cambio en sus actividades. Por ejemplo, en el caso de Luz y Roxana, ellas trabajaban en fábricas y night clubs antes de casarse con un ciudadano japonés. Luego de casarse y tener su primer hijo, tuvieron que dejar de trabajar para cuidar al recién nacido. Sin embargo, luego de dos o tres años, ambas trataban de volver a trabajar. Esto significó un proceso de negociación. Un tercer caso: cuando Roxana era soltera, trabajó por varios años en dos night clubs, donde conoció a su esposo, en ese entonces uno de los clientes. Luego él se mudó por trabajo a Shizuoka y le pidió a Roxana que dejase su trabajo y se mudara con él. Después de pensarlo, quiso renunciar, pero los empleadores no lo permitían, ya que ellos retenían su pasaporte. Roxana decidió mentirles diciendo que se iba a casar para que lo permitieran y finalmente lo consiguió. Luego de mudarse, no encontró ningún espacio para trabajar, por lo que mantuvo una relación a distancia nuevamente en Yamanashi, donde encontró otro night club. Allí también trabajaban muchas otras extranjeras con buenos beneficios laborales. Luego de tres años, su pareja fue transferida a Nagoya y le dijo «o te mudas conmigo o terminamos», por lo que ella decidió mudarse a Nagoya. No se casaron inmediatamente, ya que los padres de él no estaban de acuerdo con que su hijo se casara con una extranjera peruana. Luego de un tiempo, lo aceptaron y se casaron. Cuando su primera hija nació, los padres de su esposo la presionaron para que no trabaje y se dedique al cuidado de su hija; incluso le ofrecieron dinero por ello. Roxana afirma: «Yo quería trabajar porque ya estaba cansada de estar sola en mi casa, hasta cuando nació mi segunda hija. No sé, supongo que todo el día, todos los días, solo ver la cara de mi hija, me estresaba».

Así, a pesar de la presión de sus suegros, ella empezó a trabajar a medio tiempo en fábricas y regresaba a las 5 p.m. a cuidar a sus hijas. Eventualmente, renunció a estos trabajos y empezó a vender cosméticos por catálogo entre otras peruanas. Luego de la crisis económica de 2008, ella y Luz comenzaron a ofrecer comida 
peruana a las afueras del consulado de Perú en Nagoya. Ella tuvo la iniciativa del proyecto y fue quien capacitó a las demás. También empezó en su casa, con la ayuda de su nuera y sus hijas, un pequeño restaurante de comida peruana que atendía solo los fines de semana. Luego de dos años, su esposo enfermó de un problema cardiaco, lo que la obligó a dejar ambas iniciativas. En 2017, tiempo en el que llevé a cabo el trabajo de campo, Roxana vendía solo accesorios fuera del consulado en Nagoya, negocio menos rentable que la venta de comida, del que se había hecho cargo Luz. Roxana relata que sus negocios de comida eran para ella un logro personal, pero que prioriza el tiempo con su familia ya que, entre el trabajo de su esposo y el colegio de sus hijas, los fines de semana son el único momento que tiene para compartir con ellos.

Las historias de Luz y Roxana trabajando a medio tiempo y luego comenzando un negocio informal muestran un tipo de trayectoria de mujeres que, a pesar de que sus parejas tienen ingresos asegurados, decidieron tener una actividad adicional por motivos extraeconómicos. Además, para ellas casarse significaba más que garantizar su estatus legal: era también una negociación de sus emociones, sus sueños de tener una familia, un tipo de vida diferente del Perú en un país extranjero, trabajar y manejar una red de contactos. Luego de casarse, a pesar de ser económica y legalmente estables por la condición del esposo y su familia, buscaron desarrollar actividades económicas fuera de su trabajo no remunerado en casa. En este sentido, buscaron un trabajo en el cual pudieran estar alrededor de otras personas y entablar conexiones, aun cuando estas fueran poco duraderas. El empleo informal que se autocrearon fuera del consulado de Perú, más que una necesidad económica, es un espacio para estar en contacto con otros connacionales, especialmente con otras mujeres, hablar en español y mantener una pequeña comunidad de emociones e identidad peruana que escasea entre los peruanos en Japón, quienes están en permanente fisión.

En estas circunstancias, el tiempo que negocian para una actividad remunerada significa también la negociación de los significados que otorgan a ser madre, esposa y mujer (Fuller, 1993). En comparación con otras mujeres no migrantes que deben o desean trabajar al mismo tiempo que ser madres, las madres peruanas en Aichi se ven obligadas a hacer un balance entre, por un lado, una conexión cultural-emocional con sus hijos y su familia, y por otro, su deseo de expandir sus conexiones más allá de las familiares.

Un segundo aspecto que influye en su relación entre su trabajo, su matrimonio y sus hijos es la inestable comunidad de sus connacionales peruanos en Aichi. Los tipos de trabajo a los que se dedican estos, el ritmo de vida japonesa de 
largas jornadas laborales y la escasa formación de barrios en las zonas urbanas da poco espacio para la formación de comunidades de peruanos. Esto perjudica a las madres, ya que reproduce la desconfianza entre migrantes a los hijos de estas, quienes desarrollan anticuerpos para aprender el idioma y el bagaje cultural materno. Este potencial distanciamiento deja a sus hijos sin espacios para practicar castellano fuera del hogar. Cuando los niños no tienen un nivel aceptable de castellano, se produce el aislamiento de las madres, ya que son las únicas hispanohablantes en casa, y las deja sin recursos para sobrellevar sus limitaciones en sus roles como madre en la escuela, hospital, etc. Es por eso que, en especial las mujeres que migraron solteras, buscan crear y mantener espacios de autodesarrollo en donde puedan manejar una red de apoyo.

Por otro lado, esto puede entrar en conflicto con el tiempo que necesitan dedicar a la crianza de sus hijos y reforzar el idioma y la transmisión de códigos culturales. Lo último es especialmente importante, ya que se encuentran en desventaja frente a los estímulos que estos reciben de la cultura japonesa. Es decir, las mujeres que he citado intentan mantener una ocupación remunerada o poco remunerada fuera del hogar para crear conexiones con sus connacionales y, a la vez, insertar a sus hijos en un ambiente de habla hispana, incentivando su aprendizaje y evitando el aislamiento. Sin embargo, esta meta no siempre se cumple, ya que en la práctica se ven obligadas a negociar su tiempo entre su actividad extraeconómica y la crianza de sus hijos como dos ámbitos separados. Este tiempo de inversión entre ellas y sus hijos se vuelve hasta más importante que el que dedican sus hijos a la práctica de la escritura japonesa.

Para qué presionarlos con que estudien tanto, luego van a tener que trabajar toda su vida, aunque sea que tengan una buena niñez [...] Yo he escuchado que los niños se estresan y se vuelven locos, yo no quiero que eso les pase a mis hijas $[\ldots]$ En el futuro me gustaría abrir mi restaurante, pero ahora prefiero pasar los fines de semana con mis hijas y mi esposo; trabajar mucho no sirve, ahora mis hijas vienen y me cuentan sus cosas, eso me gusta (Roxana).

Así, si bien tratan de mantener conexiones fuera del núcleo familiar, el tiempo con sus niños es el mayor recurso que tienen para compensar el escaso apoyo hacia una educación bilingüe con la cual sus hijos puedan entablar conexiones con ellas al mismo tiempo que ascender en la sociedad japonesa manejando ambas lenguas. Como parte de la migración, las madres, escasas de conocimiento sobre Japón, se han sentido en desventaja para poder asesorar a sus niños con respecto a su mundo exterior, en este caso representado por Japón. Es decir, la 
madre entendida como madre-guía ha sido un concepto presente, pero difícil de alcanzar, por lo que han tratado de compensarlo dando prioridad al tiempo que invierten con sus hijos. En este punto, muchas de ellas se han sentido frustradas al convertirse en madres migrantes, ya que hablan de una aspiración de ser más madre-guía y menos madre-autoridad que ellas tuvieron en su infancia, pero estos deseos se vieron frustrados al sentir que no pueden enseñar y asesorar a sus hijos en la sociedad japonesa. Como dice Soraya, «cuando recién llegué a Japón, sentí que no sabía nada de la vida, que no podía responder a ninguna pregunta que me hacía mi hija. Me empecé a preguntar a mí misma cómo puedo ser mamá si no tengo nada qué enseñar».

\section{EL PRIMER HIJO Y LOS SIGUIENTES: FORMAS DIFERENCIADAS DE CRIANZA Y ADAPTACIÓN}

A lo largo de mi trabajo de campo, observé que algunas de las mujeres habían migrado a Japón siendo madres de un hijo. Muchos de estas decidieron llevarlos consigo o mandarlos traer en cuanto pudieron. Una vez en Japón, tuvieron uno o dos hijos más ${ }^{6}$. Así pues, he querido hacer énfasis en la crianza diferenciada de estos dos tipos de hijos: los primeros, nacidos en el Perú, hablaban mejor castellano que los siguientes, pero también tenían una relación diferente con la madre, en términos de crianza y conexión emocional, que los hermanos menores. Ellos juegan un rol clave como soporte emocional de la madre, y también en la crianza de los hijos menores.

\section{El papel del primer hijo: el intérprete}

En los casos de Giovana, Ana, Soraya y Jackelyn, quienes migraron siendo madres de un niño o niña y luego tuvieron uno o dos hijos más, el primer hijo o hija nacido en el Perú migró a Japón cuando tenía entre cuatro y nueve años. En este sentido, el castellano es su primera lengua y la utilizaba fluidamente en casa y en la escuela. Luego de migrar a Japón, este niño o niña pasa por un difícil proceso de adaptación. Primero, no solo debe aprender una nueva lengua e igualar el nivel de los demás niños de su clase, sino también por el retador choque cultural

6 No encontré a ninguna mujer peruana con más de tres hijos en Japón, lo que sigue la tendencia urbana actual tanto en el Perú como en Japón. 
en Japón, especialmente en la socialización con estos niños? También afrontan una difícil situación, ya que se habían creado una imagen muy diferente del país influidos por sus padres y la sociedad en general en el Perú: la de una mejor vida en un país desarrollado. Estaban llenos de muchos sentimientos positivos alrededor del viaje y de la vida en Japón. Emocionalmente, les es difícil superar ser los outsiders en la escuela, lo que influye en su aprendizaje. Todo niño en Japón debe asistir a la clase correspondiente a su edad. Los niños peruanos no son la excepción y deben ingresar a una clase donde solo se practica el japonés. Una de sus mayores limitantes es aprender a leer y escribir en este idioma, lo que trae consigo dificultades para aprender cursos como historia, literatura, etc. (Lagones, 2016).

En los casos mencionados, el primer hijo o hija nacido en el Perú se convierte en el principal intérprete y traductor de la madre, ya que oralmente maneja ambos idiomas fluidamente. En este sentido, este niño tiene un rol importante en el proceso de aprendizaje y de adaptación de la madre en Japón. En palabras de Giovana, «mi hija me ayudó con todo. Hasta me siento mal, porque ella tuvo que traducirme cosas que no eran para que lo haga una niña, cosas de adultos».

\section{El caso de Jackelyn}

Su primer hijo, Geimie, migró con ella para acompañar a su esposo cuando el niño tenía tres años. Desde ese año, hasta que él tuvo quince años, Geimie se mudó entre Huaral y Japón varias veces, ya que quería estar cerca de su madre y su padrastro, pero no se acostumbraba a la escuela en Japón. En el Perú vivía con su abuela. Cuando sus padres tuvieron un segundo hijo, él decidió, esta vez de forma definitiva, acompañarlos a cuidarlo y esforzarse en adaptarse a la escuela japonesa. Dado que Geimie cambió de país varias veces, cada llegada significaba una nueva adaptación, pero finalmente pudo manejar ambos idiomas mejor que el promedio de niños hijos de migrantes. Es por eso que solía ir con Jackelyn a todas las reuniones de padres en primaria y secundaria durante los años de escuela de su hermano menor, así como también a hospitales, la municipalidad, etc. Geimie era también quien revisaba las tareas de su hermano y lo motivaba a estudiar para continuar con la preparatoria y estudios superiores.

El esposo de Jackelyn me comentó que ella y su hijo Geimie son muy cercanos, hasta dependen el uno del otro, a diferencia de su segundo hijo, a quien

Se ha registrado y debatido extensamente sobre los problemas de bullying en las escuelas japonesas (Mino, 2006; Yoneyama, 2015). 
él considera más independiente: «Como nació aquí, tú sabes, se cree japonés, y los japoneses son más independientes. Mi hijo conoce gente en Osaka por una aplicación del celular».

Se han observado y documentado largamente prácticas de crianza en donde el hijo mayor es también cuidador de los siguientes hermanos. Esto se ha registrado sobre todo en sociedades no occidentales y rurales, donde los procesos históricos de construcción de la institución de la familia han sido distintos (Mead, 1985; Gottlieb, 2002). En este caso, no se trata de un rol ya establecido por herencia o tradición, sino más bien como una adaptación a su contexto migratorio. Cuando pregunté a las madres casadas con ciudadanos japoneses cómo sus esposos las habían ayudado a aprender el idioma, ellas dejaron de lado al esposo y mencionaron a sus hijos, especialmente al mayor, el intérprete. He mencionado la importancia del trabajo en cuidado y servicios para el aprendizaje de la lengua japonesa. Sin embargo, los hijos — más que el esposo- también aportan en este aprendizaje. Tener un niño bilingüe es una gran ventaja sobre las madres que solo tienen hijos nacidos en Japón con bajo nivel de castellano.

Pero, sobre todo, todas estas actividades que el hijo mayor lleva a cabo: intérprete, acompañante, ayudante y asesor de idiomas, lo llevan a ser parte importante de la maternidad de estas mujeres, convirtiéndose así, y más que nada, en un apoyo emocional ante el estrés de tener que adaptarse a un país tan distinto como Japón y forman parte de su proceso de construcción de maternidad como madre-guía. Así pues, ser primeriza no es solo criar a un niño por primera vez, sino que, sumado a la falta de experiencia como madre, está la falta de experiencia en el medio social japonés.

\section{EL SEGUNDO Y LOS SUBSIGUIENTES HIJOS}

«Geimie me contaba todo a mí, pero el segundo... No sabemos qué hacer. Él siempre ha sido muy discreto. Me siento a su costado, para hacerlo entender que puede confiar en mí»».

Jackelyn

En los mismos casos, Giovana, Ana, Soraya y Jackelyn ayudan a exponer mi último punto: la comparación de los segundos hijos frente a los mayores. Sus segundos y siguientes hijos nacieron en Japón y su primera socialización fue en castellano, con la madre. Sin embargo, fueron expuestos al idioma japonés desde muy temprano (tres o cuatro años de edad) en educación inicial o guardería, por lo tanto, su español es muy limitado: entienden conversaciones simples del 
día a día, pero su habilidad para expresarse es muy básica. Además, la lectura y escritura es casi nula. Ya que son fluidos en japonés, tienen menos problemas para socializar y manejan mejor los códigos culturales locales. A pesar de esto, también tienen problemas con los cursos en la escuela y la socialización con otros niños japoneses, aunque en menor medida en comparación con sus hermanos mayores nacidos en el Perú.

En la escuela, a pesar de ser fluidos en japonés, uno de sus principales problemas es aprender los kanjis (caracteres ideográficos del idioma japonés) ${ }^{8}$, sin los cuales no pueden acceder a información cada vez más compleja y abstracta. Esta dificultad se debe también a la poca asesoría que los padres les pueden brindar. Cuando estos niños crecen, su japonés se convierte en su lengua materna. Aunque muy pocos llegan al nivel universitario, al mismo tiempo cada vez hay más niños peruanos en escuelas técnicas y universidades. Por otro lado, su castellano sigue siendo muy limitado: entienden conversaciones del día a día, pero sus habilidades escritas, orales y de lectura son casi nulas. Es por esto que los segundos hijos nacidos en Japón no son buenos intérpretes para la madre. Los padres están muy orgullosos de estos niños, ya que son un símbolo del progreso de la familia en la sociedad japonesa. Por otro lado, los padres sienten que, a diferencia del primer niño o niña, el segundo hijo no valora su proceso migratorio y adaptativo ni las oportunidades por las que se han sacrificado.

Luego de su primera experiencia, las migrantes tratan de ser mejores madres con los segundos hijos en términos de poder manejarse mejor en Japón, es decir, ser mejores guías en este medio. Esto significa tener más fluidez y entendimiento del idioma, manejarse mejor en la burocracia japonesa, la escuela y los beneficios sociales que pueden ser recursos para el ascenso social de estos niños. Por otro lado, estos segundos hijos están más identificados con Japón que los primeros, lo que hace a los padres sentirse más alejados de ellos. En este sentido, no es solo la barrera del idioma lo que hace la comunicación difícil entre los padres y el segundo niño, sino que sienten que estos hijos no tienen la motivación de comunicarse con ellos acerca de sus emociones y necesidades, por lo que su conexión (bonding) madre-hijo es más difícil. Esto es especialmente importante, ya que las madres peruanas en Japón buscan compensar la débil conexión emocional entre ellas y la sociedad japonesa con la familia, especialmente con los hijos.

8 Originalmente, el sistema ideográfico de kanjis fue creado e importado de China, aunque luego simplificado para el idioma japonés. Existen más de 3000 caracteres, cada uno de los cuales expresa una idea. Tiene varias lecturas y combinaciones que forman nuevos significados. 


\section{El caso de Giovana}

«Aquí los niños son muy independientes, van solos al colegio».

Giovana

Su segundo hijo tenía trece años cuando la entrevisté. Ella le habla completamente en castellano y él contesta en un castellano limitado. A él no le gusta estudiar, pero es muy bueno en deportes: está en el equipo de fútbol de su escuela. $\mathrm{Su}$ buen manejo del idioma japonés y sus habilidades de deportista le han evitado problemas de bullying. Giovana dedica gran parte de su tiempo a llevarlo y traerlo de sus entrenamientos, cocinarle, etc. Tiene un trabajo de medio tiempo en una empresa de remesas de Japón a Perú, por lo que también frecuenta el consulado peruano y a las peruanas que venden viandas fuera de este. Su segundo hijo no tiene problemas para interactuar con otros jóvenes de su edad, pero en palabras de Giovana, ella cree que su hijo se siente más cómodo con otros niños extranjeros. Además, afirma que él no la puede ayudar a traducir del japonés al castellano, porque se le dificulta explicar varios conceptos japoneses, a diferencia de su primera hija. Él muestra ser independiente de muchas formas, entre ellas, rehusar al cariño físico de Giovana, lo que ella ha tenido que negociar por muchos años. Dedicar tiempo a dejarlo en el colegio y en sus entrenamientos es una forma de mantener esta conexión, a pesar de que no sea lo usual entre padres japoneses.

Finalmente, las madres tienen diferentes relaciones con los primeros hijos nacidos en el Perú, y los segundos, nacidos en Japón. Sin embargo, ambos son una motivación para las madres para manejar diferentes recursos culturales en Japón y, en algunos casos, para la búsqueda de algún tipo de desarrollo personal.

\section{CONCLUSIONES}

A lo largo del seguimiento de los procesos migratorios de este grupo de mujeres, abordé sus motivaciones para migrar, cambios con respecto a su situación laboral y su estado civil, y finalmente, la relación diferenciada que establecen en la crianza de sus hijos. Considero que existen cuatro elementos principales entre los cuales las mujeres reconceptualizan la maternidad en Japón: la migración, la casa, la escuela y el trabajo. Considero que su proceso migratorio es transversal a todos los demás elementos. Es decir, no podemos analizar ninguno de los demás elementos sin pensar en el proceso migratorio. Por su parte, el elemento de la casa entra en tensión con los otros dos: la escuela y el trabajo, de modo que en 
un gráfico tendría un lugar central, mientras que la escuela y el trabajo ocupan los espacios laterales y (en este análisis) no guardan una relación directa.

La tensión entre la casa y el trabajo está relacionada con lo que la sociedad japonesa les exige en un plano estructural: el rol de madre como una mujer encargada exclusivamente de los intereses de la familia, rol apoyado por el sistema de impuestos que ejerce presión sobre las parejas para que solo uno de sus miembros tenga un trabajo remunerado (Nakamoto, 2014; Wingfield-Hayes, 2013). Por otro lado, en el plano subjetivo, las mujeres peruanas - en especial las que migraron solteras - construyeron un imaginario de la vida en Japón en donde podrían tener acceso a un tipo de ascenso social no por medio de la educación superior, sino basado en vivir en el extranjero y ser una mujer de mundo, lo que se traduce en lo cotidiano en el acceso a un consumo diferente que en el Perú, y tener un trabajo que les permitiera construir redes y no ser exclusivamente amas de casa. En el caso de los peruanos estudiados en la prefectura de Aichi, esto tiene especial relevancia, ya que no se puede afirmar que haya una «guetificación» de peruanos en esta región, a diferencia de los casos de peruanos en otros países desarrollados, como Estados Unidos o España (Altamirano, 2009; Berg y Ramos Zayas, 2015). Por ello, sus conexiones son débiles, lo que las deja en un potencial aislamiento que tratan de evitar a través de actividades económicas que les permite mantener conexiones con connacionales.

Esta tensión entre el trabajo y la casa también involucra su conexión con sus hijos, quienes son una principal fuente de conexión de connacionales. Si bien por medio del trabajo desarrollan recursos para poder llegar a ejercer una maternidad guía — es decir, desenvolverse mejor en el medio japonés para guiar a los hijos en él-, es también una limitación para una crianza que busca insertar a sus hijos como sujetos dentro de su bagaje cultural como peruanas viviendo en Japón.

En cuanto a la casa, esta es el ámbito privado de interacción directa entre la madre y los hijos, en donde se transmiten las estructuras familiares - y, por extensión, las culturales_-; es el espacio en donde las madres peruanas pueden llevar a cabo su función ideal de madre guía en oposición a la escuela, que transmite saberes formales. En el caso de las madres peruanas en Japón, esta división es conflictiva, ya que la escuela termina inevitablemente transmitiendo y socializando al niño en el ámbito japonés. La escuela les permite desarrollar lo llamado por Yamashiro como «cultural literacy» o lectura y desarrollo de códigos culturales ${ }^{9}$ como parte

\footnotetext{
$9 \quad$ Traducción del autor.
} 
de la identidad con la que se construyó el Estado-nación japonés (Yamashiro, 2013; Iwabuchi, 2002).

A su vez, las posibilidades de ascenso social dependen de la educación (Takenaka, 2010). Las madres apoyan este aprendizaje, pero tienen el ideal de tener hijos bilingües y, por extensión, que compartan también sus propios códigos culturales. Al no tener acceso a escuelas bilingües español-japonés, tienen dos opciones: ser ellas las maestras, o que los niños lleven algún curso por internet en el cual ellas también deban ser tutoras. En ambos casos, ser madre-profesora es una relación nueva que no todas están dispuestas a asumir. Con relación a lo que dice Roberta Raffaeta sobre el actual contexto de la educación global, para ser buena madre en nuestros tiempos es necesario tener ciertos saberes especializados, especialmente en pedagogía y psicología (2016). Por tanto, las poblaciones vulnerables se encuentran en mayor desventaja aún. Ese es el caso de las peruanas en Japón: el hecho de convertirse en madre-profesora o madre-tutora demanda tiempo y esfuerzo, del cual no todas disponen. Si bien la escuela ha jugado un rol importante ayudando a las madres a criar a sus hijos, dejándoles tiempo para diversificar sus actividades laborales, en este contexto de migración y necesidad de bilingüismo, el formato de la educación en una escuela juega en su contra.

Con relación a estas demandas de maternidad y crianza actuales, considero que las madres peruanas de mi muestra forman parte de un proceso global en donde la buena maternidad se relaciona no solo con actos, sino más bien con actitudes de diálogo más que de imposición (Raffaeta, 2016). Sin embargo, estos procesos se complejizan en el contexto migratorio. En el caso de las madres peruanas en la prefectura de Aichi, Japón, su situación migratoria dificulta su ideal de madreguía, ya que, como muchas poblaciones de migrantes, carecen de varios recursos en comparación con la población local. Sin embargo, las peruanas en Japón se enfrentan no solo a la alteridad cultural en donde la conexión con sus hijos está en juego, sino también la carencia de una comunidad de pares connacionales que sirva de apoyo a una crianza de niños con menos brechas culturales con sus madres, y además, sujetos que crezcan en interculturalidad, que conecten con sus madres, pero también reproduzcan comunidad que sea una red de apoyo emocional.

Finalmente, la pregunta de cómo reconceptualizan la maternidad está conectada con la pregunta de qué sujetos esperan insertar a la sociedad y a qué tipo de sociedad: una dividida entre la hegemonía de la cultura japonesa y grupos de migrantes emergentes, o una en donde sean sujetos que aporten al diálogo intercultural en donde la alteridad sea tomada no solo como un constante reto, sino como un aporte. Respecto de esto, pensar en las madres y sus hijos como 
un elemento aislado no sería suficiente para entender el fenómeno de cambio en la construcción de la maternidad. Así, pues, mi etnografía busca dar cuenta de la carga social que se atribuye a la maternidad como un elemento central en la reproducción y transformación social en un contexto migratorio.

\section{REFERENCIAS}

Altamirano, Teófilo (14 noviembre 2009). Los peruanos en el exterior y su revinculación con el Perú. https://dejacontarte.files.wordpress.com/2009/11/altamirano1.pdf

Ames, Patricia (2014). Cambios en las estrategias de vida y en la demografia de las jovenes mujeres rurales. En A. Diez, E. Raez y R. Fort (eds.), Perú: el problema agrario en debate (pp. 86-113). Lima: Sepia.

Anderson, Jeanine (2012). La migración femenina peruana en las cadenas globales de cuidados en Chile y España. Tranferencia de cuidados y desigualdad de género. Lima: ONU mujeres.

Berg, Ulla y Ana Y. Ramos Zayas (2015). Racializing Affect. A theoretical Preposition. Current Anthropology, 56(5), 654-676. https://doi.org/10.1086/683053

Brettel, Caroline B. (2008). Theorizing Migration in Anthropology. The social Construction of Networks, Identities, Communities and Globalscapes. En Migration Theory: Talking about Disciplines (pp. 113-159). Nueva York: Routledge.

Fritzs, Barbara, Ingrid Kummels y Stefan Rinke (2013). Migration between spaces in the Americas and Beyond. The Journal of the International Association of InterAmerican Research, 6(2), September.

Fuller, Norma (1993). Dilemas de la femineidad: mujeres de clase media en Perú. Lima: PUCP.

Fuller, Norma y Mara Viveros (2001). Paternidades en América Latina, un estudio comparativo entre Lima y Bogotá. Ciudad: PRODIR, Informe de Investigación Programa de Derechos Reproductivos, Fundación Carlos Chagas.

Gottlieb, Alma (2002). New Developments in the Anthropology of Childcare. Anthropology News, 43(7), 13. https://doi.org/10.1111/an.2002.43.7.13

Hernández, Berenice (2006). Nuevas rutas, nuevas actoras: mujeres migrantes latinoamericanas en Alemania. En M. I. Garcia Gossio, Los nuevos escenarios de la migración: causas, condiciones, consecuencias. México, D. F.: Fundacion Henirich Boll.

Hernández, Berenice (2007). ¡Pues para Europa! La migracion latinoamericana a Alemania desde una mirada de género. En I. Yépez del Castillo y G. Herrera 
(eds.), Nuevas migraciones latinoamericanas a Europa, balances y desafios. Barcelona: Obreal.

INEI (2008). Perú: estadísticas de la emigración internacional de peruanos e inmigración de extranjeros 1990-2007. Lima: Cooperacion Interinstitucional INEI, Digemin, OIM.

INEI (2013). Perú: estadísticas de la emigración internacional de peruanos e inmigración de extranjeros 1990-2012. Lima.

INEI (2017). Evolución del movimiento migratorio peruano junio 2017. Informe técnico. Lima.

Iwabuchi, Koichi (2002). «Soft» Nationalism and Narcissism: Japanese popular culture goes global. Assian Studies Review, 26(4), 447-469. https://doi. org/10.1080/10357820208713357

Lagones, Jakeline (2016). Challenge of Japanese-Peruvian descendent families in the XXI century. Doctoral Thesis. Nagoya University.

Lauser, A. (2008). Philippines Women on the move: Marriage across borders. International Migration, International Organization for Migration (IOM), 46(4), 85-110. https://doi.org/10.1111/j.1468-2435.2008.00473.x

Mahler, Sarah J. y Patricia R. Pessar (2006). Gender matters: Ethnographers bring gender from the periphery toward the core of migration studies. International Migration Review, 40(1), 27-63. https://doi.org/10.1111/j.1747-7379.2006.00002.x

Mead, Margaret (1985). Adolescencia, sexo y cultura en Samoa. México, D. F.: Origen/ Planeta.

Melgar Tisoc, Dahil M. (2014). En los márgenes del Sol Naciente. Etnicidad, violencia y pertenencias en la migración de peruanos en Japón. Tesis de maestría en Antropología Social. México, D. F.: Ciesas.

Mino, Tamaki (24-25 febrero, 2006). Ijime (Bullying) in Japanese Schools: A Product of Japanese Education Based on Group Conformity. Rhizomes: Re-Visioning Boundaries, School of Languages and Comparative Cultural Studies, The University of Queensland. shorturl.at/jlmsD

Moorehead, Robert (2007). Teaching and Learning Across an Ethnic Divide: Peruvian Parents and a Japanese School. Okinawa: University of the Ryukyus Repository.

Moorehead, Robert (2010). You can't go home again: Japanese Peruvian Inmigrants and the Struggle for Integration and Identity in the Japanese homeland. Dissertation. Doctor of Philosophy in Sociology. University of California.

Nakamoto, Atsushi (2014). Japan's income tax base: comparison with other countries and estimation of Tax Reform. Policy Research Institute, Ministry of Finance. Japan. Public Policy Review, 10(3), 397-413. 
Nakamura, Patricia (2011). La inclusión de las familias inmigrantes en la escuela: el caso de los peruanos en Japón. Revista Iberoamericana sobre Calidad, Eficacia y Cambio en Educación, 9(4), 158-173.

Olavarría, José (2017). Sobre hombres y masculinidades. Santiago de Chile: Universidad Académica de Humanismo Cristiano.

Palomar, Cristina (2005). Maternidad, historia y cultura. La ventana Revista de Estudios de Género. Mexico: Universidad de Guadalajara.

Raffaeta, Roberta (2016). Migration and Parenting: reviwing the debate and calling for future research. International Journal of Migration Health and Social Care, 1, vol. 12. Emerald Group. https://doi.org/10.1108/IJMHSC-12-2014-0052

Samman, E., E. Prestel-Marshall, N. Jones, T. Bhatkal, C. Melamed, M. Stavropoulou y J. Wallace (2016). Women's work. Mothers, children and the global childcare crisis. Report 2016. Londres: Overseas Development Institute.

Takenaka, Ayumi (2010). How ethnic minorities experience social mobility in Japan: An ethnographic study of Peruvian Migrants. En H. Ichida y D. Slater (eds.), Social Class in Contemporary Japan. Structures, Sorting and Strategies (pp. 221-238). Nueva York: Routledge.

Weisner, Thomas S. (2015). Childhood Anthropological Aspects. En J. D. Wright (ed.), International Encyclopedia of the Social \& Behavioral Sciences (pp. 451-458). Oxford: Elsevier. https://doi.org/10.1016/B978-0-08-097086-8.12032-X

Weiss, Nancy P. (1978). The Mother-Child Dyad Revisited: Perceptions of Mothers and Children in Twentieth Century Child-Rearing Manuals. The Journal of Social Issues, 29-45. https://doi.org/10.1111/j.1540-4560.1978.tb01027.x

Wiltshire, Rosina (1992). Implications of Transnational Migration for Nationalism: The Caribean example. En Towards a Transnational perspective of migration: Race, class, ethnicity and Nationalism (pp. 175-187). Nueva York: New York Academy of Science. https://doi.org/10.1111/j.1749-6632.1992.tb33491.x

Wingfield-Hayes, Rupert (2013). Japan: The worst developed country for working mothers? Tokio: BBC News.

Yamashiro, Jane H. (2013). The Social Construction of Race and Minorities in Japan. Sociology Compass, 7(2), 147-161. https://doi.org/10.1111/soc4.12013

Yoneyama, Shoko (2015). Theorizing school Bullying: insights from Japan. Confero, 3(2), 120-160. https://doi.org/10.3384/confero.2001-4562.150628 